\title{
Hermafroditismo verdadeiro na espécie canina
}

\author{
True Hermaphroditism in a Dog \\ Paula Priscila Correia Costa', Cleyson Teófilo Braga Filho², Luana Azevedo de Freitas ${ }^{2}$, Maressa Holanda dos \\ Santos ${ }^{2}$, Leonardo Alves Rodrigues Cabral ${ }^{2}$, João Alison de Morais Silveira ${ }^{3}$ \& Airton Alencar de Araújo'
}

\begin{abstract}
Background: Hermaphroditism or intersex is a general term that includes various congenital anomalies of the genital system which is used to define animals with ambiguous sexual characteristics. It occurs in domestic animals, more commonly in pigs and goats, and rarely in horses, dogs, sheep, and cattle. The prevalence of hermaphroditism varies a lot among breeds and species and is higher in groups with a high degree of consanguinity. Therefore, the objective of this report is to describe a case of canine hermaphroditism in a dog with male phenotype, as well as the anatomical and hormonal findings, and classification of the hermaphroditism exhibited by the animal studied.

Case: A 1-year-old, mongrel, $5 \mathrm{~kg}$ dog was referred to the UHV-UECE due to the presence of a slit on the lower quadrant of the abdomen, caudal to the umbilical scar. At examination, the animal exhibited normal rectal temperature, no alterations of palpable lymph nodes, and a satisfactory body condition score. The pubic area had 2 testicles, each one in a different scrotum, 1 to the right and 1 to the left of the slit. A prepuce with no apparent function was present cranially to the slit, closer to the umbilical scar. At the other extremity of the slit, on the pubic region, there was a flaccid structure similar to a penis (micropenis) with no penile bone and no function. The slit was open until the area ventral to the anus, where the urethra was detected. The animal exhibited a behavior of territory demarcation with urine typically seen in male dogs. Orchiectomy and slit correction surgery were performed. Pre-surgical exams included: complete blood count and hormonal doses of estradiol, testosterone, and progesterone. Abdominal ultrasound was performed to assess presence of sexual glands and gonads. The hormonal exam revealed: estradiol $56.39 \mathrm{pg} / \mathrm{mL}$; testosterone $127.9 \mathrm{ng} / \mathrm{mL}$; progesterone 0.892 $\mathrm{ng} / \mathrm{mL}$. A uterus was not detected on ultrasound examination. Ovaries were seen on their typical anatomical position; they were symmetrical and had normal sizes. There was a normal size prostate in the pelvic area, exhibiting normal texture and echogenicity. No other abnormalities were seen and the owner opted for no further surgical intervention.

Discussion: Testosterone predominance explains the male behavior and appearance, demonstrating that the testis were prevalent over the ovaries. The occurrence of XX males has been reported. The genetic cause is the absence of the SRY chromosome, which has a fundamental role on activation of the SOX gene, which is responsible for sex determination. Clinically, a true hermaphrodite can exhibit different degrees of genital ambiguity; they can be diagnosed during puberty with the emergence of heterosexual characteristics, or as an adult, with infertility or gonadal neoplasia. True hermaphrodites are individuals with testicular and ovarian tissues, either combined in one gonad (ovotestis) or present as two separate gonads. The presence of ovaries and testicles can be confirmed by histology, which was not performed in this study. However, ultrasound findings (prostate and ovaries), and the presence of normal testis and external genitalia without a defined penis or vulva are in accordance with the description of a true hermaphrodite.
\end{abstract}

Keywords: hermaphroditism, dogs, congenital anomaly.

Descritores: hermafroditismo, cães, anomalia congênita. 


\section{INTRODUÇÃO}

Hermafroditismo ou intersexo é um termo geral que inclui várias anomalias congênitas do sistema genital, usado para definir animais que apresentam características sexuais ambíguas [15]. Sob a denominação de intersexo estão enquadrados os hermafroditas verdadeiros, os pseudo-hermafroditas e outras formas de reversão sexual [15].

Os intersexos e as várias anomalias relacionadas ao aparelho reprodutor ocorrem nos animais domésticos [19] sendo mais frequentes em suínos e caprinos e raramente verificados em equinos, cães, ovinos e bovinos [11], mas sua prevalência varia grandemente entre as linhagens, raças e espécies, sendo muito maior naquelas em que há alto grau de cruzamentos consanguíneos, por escolha ou por falta de machos mantidos como reprodutores [12].

Além do diagnóstico clínico por palpação retal e ultrassonografia, em que as anomalias podem ser detectadas [9], o diagnóstico exige a constatação histológica de tecido ovariano e testicular [16].

O tratamento clínico (reposição hormonal) e cirúrgico com correção da genitália interna e externa vai depender da opção do sexo de criação a ser definida pela equipe médica em conjunto com o proprietário do animal [2].

O presente relato tem como objetivo abordar um caso de hermafroditismo canino com fenótipo masculino, descrever os achados anatômicos encontrados e avaliar os níveis hormonais encontrados para classificação do tipo de hermafroditismo apresentado pelo animal em estudo.

\section{CASO}

Foi atendido, na Unidade Hospitalar Veterinária (UHV) da Universidade Estadual do Ceará (UECE), um cão, SRD, 1 ano de idade, com $5 \mathrm{~kg}$. O proprietário levou o cão devido a uma fenda caudal a cicatriz umbilical, no quadrante inferior do abdômen (Figura 1).

Foi realizado um exame físico e clínico. No exame clínico o animal apresentou temperatura retal normal, mucosas normocoradas, tempo de preenchimento capilar (TPC) normal, linfonodos não palpáveis e escore corporal satisfatório. Na região pubiana foi possível constatar dois testículos, cada um em um saco escrotal diferente, um à direita e outro à esquerda da fenda. Na região anterior a fenda, mais próximo à cicatriz umbilical, foi detectada a presença de prepúcio, sem função aparente. Na outra extremidade da fenda, na região pubiana, havia a presença de uma formação semelhante ao pênis (micropênis), flácida e sem presença de osso peniano, também sem função. A fenda persistia até a região logo abaixo do ânus, onde foi possível detectar a uretra (Figura 2). No consultório o animal apresentava comportamento masculino, com o ato de demarcar território através da urina.

Para a correção da fenda, optou-se por uma cirurgia corretiva e realização de orquiectomia (Figura 3). Para tanto, foi solicitado hemograma completo e dosagem hormonal de estradiol, testosterona e progesterona. Posteriormente foi feita uma ultrassonografia abdominal para verificar presença de glândulas e gônadas sexuais.

O hemograma completo apresentou valores dentro da normalidade para a espécie. O exame hormonal apresentou o seguinte resultado: estradiol, $56,39 \mathrm{pg} / \mathrm{mL}$; testosterona $127,9 \mathrm{ng} / \mathrm{mL}$; progesterona $0,892 \mathrm{ng} / \mathrm{mL}$.

De acordo com o laudo da ultrassonografia, não foi visualizado útero. Foram detectados ovários em topografia padrão, simétricos e de tamanho normal, esquerdo 1,13 cm (Figura 4A) e direito $1,33 \mathrm{~cm}$ (Figura 4B) [corte longitudinal] e próstata em localização pélvica, de tamanho normal (Figura 4C) [distância entre lobos em corte longitudinal de 1,65 cm]. Parênquima normoecóico e textura normal.

$\mathrm{O}$ animal retornou para retirada dos pontos. Não apresentava nenhuma outra anormalidade e o proprietário optou por não realizar nenhuma intervenção cirúrgica a mais.

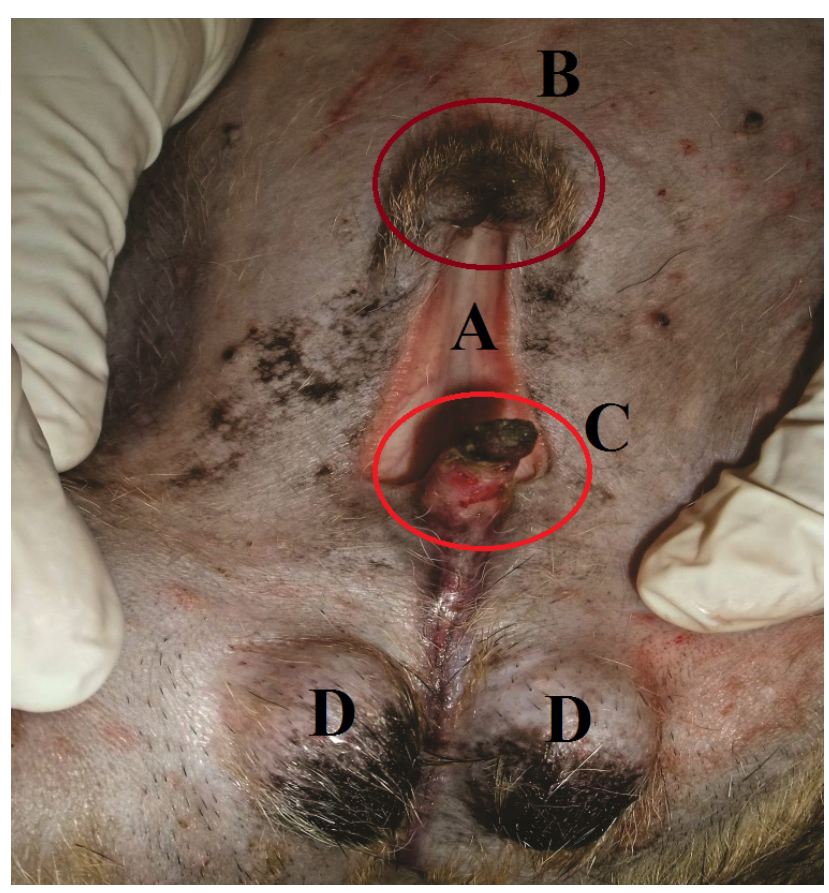

Figura 1. A- Fenda na região abdominal inferior. B- Prepúcio na região anterior. C- Pênis afuncional na região anterior. D- Dois testículos individualizados, cada um em um saco escrotal. 


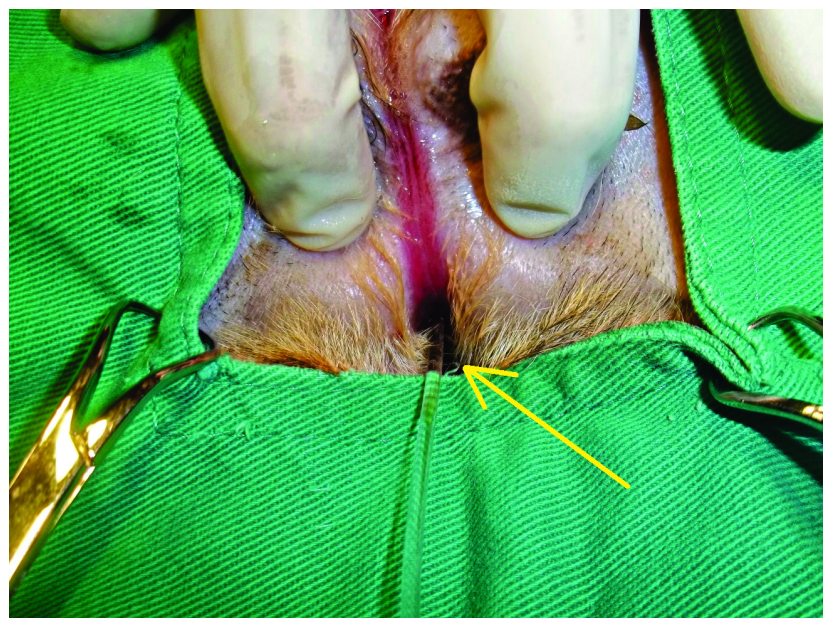

Figura 2. Sonda uretral indicando localização da uretra, usada para averiguar anormalidades na uretra (seta amarela).

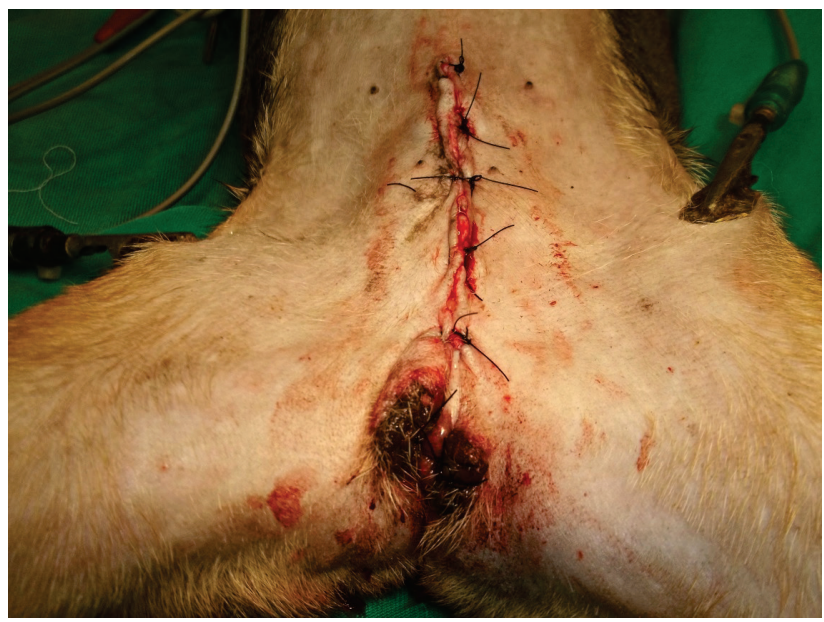

Figura 3. Correção da fenda, retirada das estruturas afuncionais e orquiectomia total.

\section{DISCUSSÃO}

Os níveis hormonais encontrados revelam níveis de testosterona predominantes sobre os níveis de estrógenos e progesterona. A predominância da testosterona explica o fato do animal apresentar aspecto e comportamento masculino, demonstrando, assim, que o testículo predominou sobre o ovário existente. Todavia, diante dos valores de estradiol e progestero$\mathrm{na}(56,39 \mathrm{pg} / \mathrm{mL}$ e $0,892 \mathrm{ng} / \mathrm{mL})$ respectivamente,o animal foi considerado em proestro.

O principal evento hormonal durante o proestro é o aumento contínuo da concentração de estradiol sérico. O início do proestro está associado a concentrações de estrógeno acima de $25 \mathrm{pg} / \mathrm{mL}$, atingindo o pico máximo com concentrações em torno de 60 a 70 pg/mL, 24 a $48 \mathrm{~h}$ antes do término do mesmo [5]. Por conseguinte, a progesterona é produzida pelas células da granulosa nos folículos maduros que so- frem luteinização sob influência do LH [1] antes do estro. As concentrações circulantes de progesterona encontram-se basais $(<0,5 \mathrm{ng} / \mathrm{mL})$ até 24 a $72 \mathrm{~h}$ antes do final do proestro, quando se eleva acima de 1,0 $\mathrm{ng} / \mathrm{mL}$ [5]. Desta forma, a presença e a concentração sérica dos hormônios supracitados corrobora com os

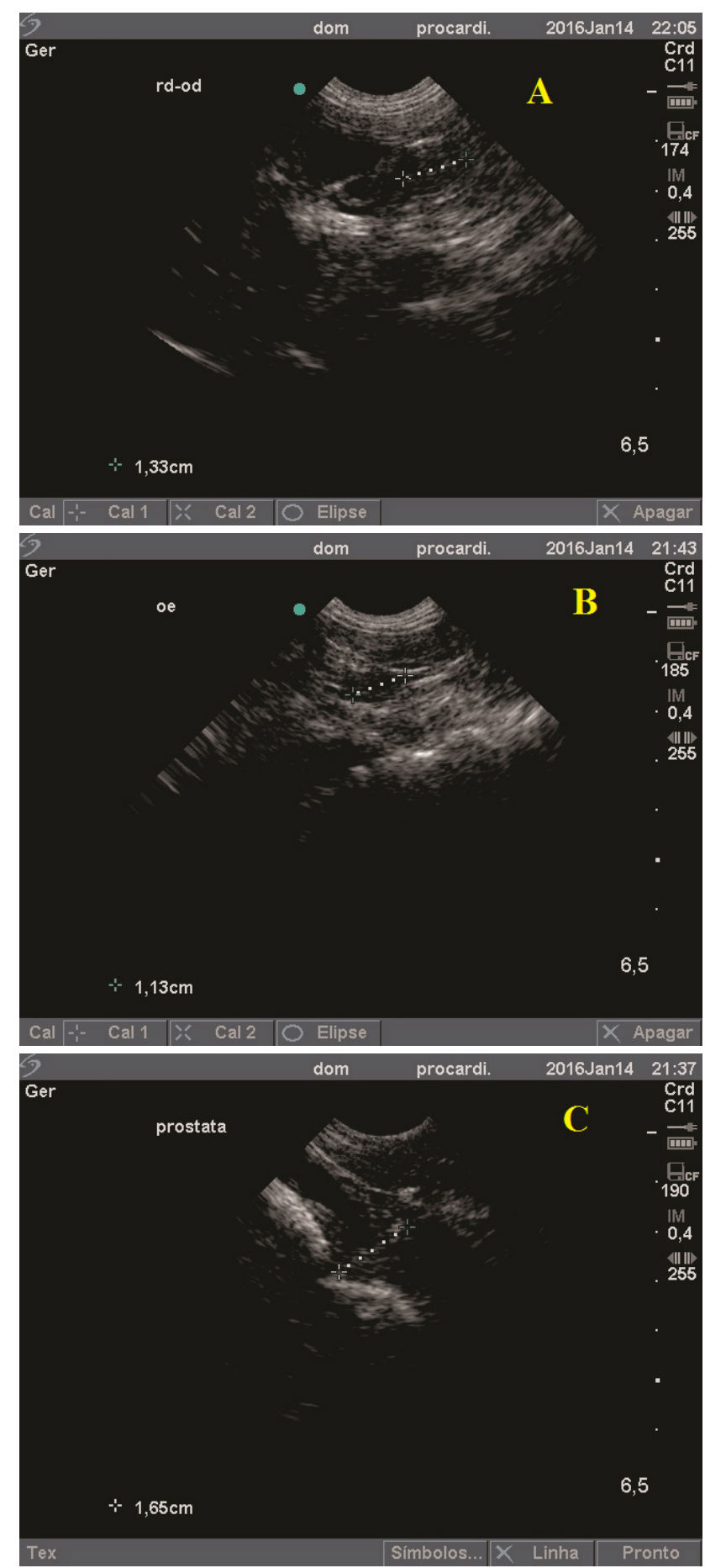

Figura 4. Imagem ultrassonográfica. A- Traço pontilhado indicando localização e comprimento $(1,33 \mathrm{~cm})$ do ovário direito (od), corte longitudinal. B- Traço pontilhado indicando localização e comprimento $(1,13 \mathrm{~cm})$ do ovário esquerdo (oe), corte longitudinal. C- Traço pontilhado indicando localização e comprimento $(1,65 \mathrm{~cm})$ da próstata. 
achados ultra-sonográficos de gônadas femininas e funcionais (ovários).

Em animais pseudo-hermafroditas masculino ou com persistência do ducto de Müller, é esperado que se encontre genitália externa masculina, com presença de osso peniano, criptorquidismo uni ou bilateral e dois cornos uterinos completos. O mesmo ocorre com o pseudo-hermafrodita feminino, onde se observa genitália feminina com estruturas internas masculinas [13]. O que não é compatível com o animal em questão, já que na ultrassonografia foi possível detectar próstata, ovários e os dois testículos não se mostraram atrofiados, sendo anatomicamente normais.

Neste estudo de caso não foi possível realizar o exame citogenético do animal para conhecimento do sexo genético, visto que não se dispõe na região de laboratório especifico para tal exame, o que seria importante para verificar a coincidência do sexo fenótipo com o genético [12].

Vários autores relatam em cães a ocorrência de machos XX [6,10]. A causa genética desta desordem está na ausência do cromossomo SRY, que tem papel fundamental na ativação do gene SOX responsável pela determinação do sexo [4,17].

Os hermafroditas verdadeiros possuem cariótipo variável, sendo a maioria de cariótipo feminino normal e aqueles que possuem cariótipo XX/XY são provavelmente quimeras, que se originam de dupla fertilização ou quando ocorre a anastomose vascular após ocorrer fusão das membranas fetais de embriões machos e fêmeas [7].

Clinicamente o hermafrodita verdadeiro pode se apresentar com os mais variados graus de ambiguidade genital, ou, até mesmo, durante a puberdade, com o apa- recimento de características heterossexuais, ou ainda, na vida adulta, com infertilidade ou neoplasia gonadal $[3,7]$.

Hermafroditas verdadeiros possuem tanto tecido ovariano quanto tecido testicular presentes em seu organismo $[8,14,18]$. Os hermafroditas verdadeiros são indivíduos com tecido testicular e ovariano ou combinados em uma única gônada (ovotestis) ou existentes em gônadas separadas [2].

A presença de ovários e testículos só pode ser confirmada através de exame histológico, que não foi feito neste trabalho, no entanto os achados ultrassonográficos (próstata e ovários), bem como a constituição anatômica normal dos testículos, e genitália externa com ausência de pênis ou vulva definida estão de acordo com a descrição para a classificação de hermafrodita verdadeiro [16].

A classificação da intersexualidade pode ser com base na presença das gônadas existentes e de estruturas ambíguas e rudimentares do trato genital; assim temos o hermafroditismo ovariano, testicular e hermafroditismo verdadeiro com presenças de ovários e testículos no mesmo indivíduo, associados à presença de estruturas ambíguas não bem definidas do trato genital [19]. Além de valores hormonais femininos condizentes com fêmeas em proestro, demonstrando ovários funcionais, corroboram com a classificação do animal em estudo como hermafrodita verdadeiro.

Declaration of interest. The authors report no conflicts of interest. The authors alone are responsible for the content and writing of the paper.

\section{REFERENCES}

1 Allen WE. 1995. Fertilidade e obstetrícia no cão. São Paulo: Varela, 197p.

2 Bearden H.J. \& Fuquay J.W. 2000. Anatomical and inherited causes of reproductive failure. In: Applied animal Reproduction. 5th edn. Saddle River: Prentice Hall, pp.319-327.

3 Birchard S.J. \& Sherding R.G. 1998. Manual Saunders - Clínica de Pequenos Animais. São Paulo: Roca, 1618p.

4 Chaboissier M.C., Kobayashi A., Vidal V.I., Lutzkendorf S., van de Kant H.J., Wegner M., de Rooij D.G., Behringer

R.R. \& Schedl A. 2004. Functional analysis of Sox8 and Sox9 during sex determination in the mouse. Development 131: 1891-1901.

5 Feldman E.C. \& Nelson R.W. 2003. Canine and feline endocrinology and reproduction. 3rd edn. Philadelphia: W.B. Saunders, 1089p.

6 Grunert E., Birgel E.H., Vale W.G. \& Birgel Junior E.H. 2005. Patologia e clínica da reprodução dos animais domésticos: ginecologia. São Paulo: Varela, 551p.

7 Halnan C.R.E. 1989. Cytogenetics of animals. Wallingford: CAB International, 519p.

8 Hoskins J.D. 2004. Defeitos Congênitos do Cão. In.: Ettinger S.J. \& Feldman E.C. (Eds). Tratado de Medicina Interna de Pequenos Animais - Doenças de Cão e do Gato. 5.ed. Rio de Janeiro: Guanabara, pp.2087-2101. 
9 Knobee M.G., Maenhoudt C., Turner R.M. \& McDonnell S.M. 2011. Physical, behavioral, endocrinologic, and cytogenetic evaluation of two Standard bred racehorses competing as mares with an intersex condition and high postrace serum testosterone concentrations. Journal of the American Veterinary Medical Association. 238(6): 751-754

10 Krob G., Braun A. \& Kuhnle U. 1993. True hermaphroditism: geographical distribution, clinical findings, chromosomes and gonadal histology. European Journal of Pediatric. 153: 2-10.

11 Leal S.S., ObaE., Prestes N.C., Vianna F.P., Beier S.L., Melero F.H., Zanini M. \& Motta L.S. 2002. Pseudohermafrodita masculino canino: relato de caso. In: II Congresso Paulista De Clínicos Veterinários De Pequenos Animais (São Paulo, Brasil). p.124.

12 López F.J.P., Chávari A.C.T., Dibbern S.L., González H.S., Ramos A.A. \& Nájera M.J.F. 2015. Intersexualidade em caprinos. Revista Eletrônica Veterinária. 16(6): 1-13. [Fonte:<https://www.researchgate.net/publication/281123871_ Intersexuality_in_goats>].

13 Memon M.A. \& Mickelsen D.W. 2004. Distúrbios Hereditários e Congênitos dos Sistemas Reprodutivos do Macho e da Fêmea. In: Ettinger S.J. \& Feldman E.C. (Eds). Tratado de Medicina Interna de Pequenos Animais - Doenças de Cão e do Gato. 5.ed. Rio de Janeiro: Guanabara, pp.1665-1670.

14 Nemzek J.A., Homco L.D., Wheaton L.G. \& Grman G.L. 1992. Cistic ovaries and hyperestrogenism in a canine female pseudohermaphrodite. Journal of American Animal Hospital Association. 28: 402-406.

15 Nowackan J., Nizanskin W., Klimowicz M., Dzimirad S. \& Switonskis M. 2005. Lack of the SOX9 Gene Polymorphism in Sex Reversal Dogs (78,XX; SRY negative). Journal of Heredity. 96(7): 797-802.

16 Paulino F.O., Goldshimitid B., Souza L.M. \& Mendonça M.A.S. 2001. Estudo citogenético em cão pseudohermafrodita masculino da raça Akita. Revista Brasileira de Reprodução Animal. 25: 491-492.

17 Santos J.A. Patologia Especial dos Animais Domésticos. 2.ed. Rio de Janeiro: Interamericana, 576p.

18Sutton E., Hughes J., White S., Sekido R., Tan J. Arboleda V., Rogers N., Knower K., Rowley L., Eyre H., Rizzoti K., McAninch D., Goncalves J., Slee J., Turbitt E., Bruno D., Bengtsson H., Harley V., Vilain E., Sinclair A., Lovell-Badge R. \& Thomas P. 2011. Identification of SOX3 as an XX male sex reversal gene in mice and humans. Journal of Clinical Investigation 121(1): 328-341.

19 Ticianelli J.S., Oliveira B.M.M., Zogno M.A., Arruda R.P. \& Celeghini E.C.C. 2011. Intersex and others development anomalies of the reproductive tract in the domestic animals and the cytogenetic as ancillary test to diagnosis. Revista Brasileira de Reprodução Animal. (Belo Horizonte). 35(1): 26-32. 